\title{
The Importance of Self-excitation in Spiking Neural Networks Evolved to Recognize Temporal Patterns
}

\author{
Muhammad Yaqoob[0000-0001-9328-2593] ${ }^{1}$, Volker \\ Steuber[0000-0003-0186-3580 ${ }^{2}$ and Borys Wróbel[0000-0002-4759-725X]* 1 \\ ${ }^{1}$ Evolving Systems Laboratory, Adam Mickiewicz University in Poznan, Poland \\ ${ }^{2}$ University of Hertfordshire, Hatfield, UK \\ \{yaqoob, wrobel\}@evosys.org \\ v.steuber@herts.ac.uk
}

\begin{abstract}
Biological and artificial spiking neural networks process information by changing their states in response to the temporal patterns of input and of the activity of the network itself. Here we analyse very small networks, evolved to recognize three signals in a specific pattern $(\mathrm{ABC})$ in a continuous temporal stream of signals (...CABCACB...). This task can be accomplished by networks with just four neurons (three interneurons and one output). We show that evolving the networks in the presence of noise and variation of the intervals of silence between signals biases the solutions towards networks that can maintain their states (a form of memory), while the majority of networks evolved without variable intervals between signals cannot do so. We demonstrate that in most networks, the evolutionary process leads to the presence of superfluous connections that can be pruned without affecting the ability of the networks to perform the task and, if the unpruned network can maintain memory, so does the pruned network. We then analyse how these small networks can perform their tasks, using a paradigm of finite state transducers. This analysis shows that self-excitatory loops (autapses) in these networks are crucial for both the recognition of the pattern and for memory maintenance.
\end{abstract}

Keywords: temporal pattern recognition, spiking neural networks, exloops, self-loops, artificial evolution, minimal cognition, complex networks, genetic algorithm, finite state transducer

\section{Introduction}

The current understanding of information processing in biological brains postulates that this processing is accomplished thanks to constant transitions of biological networks from one pattern of spiking activity to another $[1,3,5,11$, $12,15,23]$. Temporal input patterns in all sensory modalities, including smell [25], sight [34], and hearing [14], influence these patterns of activity; and the patterns of neural activity determine the animal behaviour. One of the central 
problems in neuroscience is how biological neural circuits can accomplish such temporal processing. Answering this question may help in designing bio-inspired artificial cognitive systems. Of special interest is how this processing, which also involves the maintenance of the spiking activity (a form of memory), while depending on the precise timing of spikes, can be accomplished in the presence of noise $[8,10]$; and indeed may necessitate noise $[6,13,29,37]$.

In this work, we analyse very small spiking neural networks (SNNs) evolved to perform a simple temporal pattern recognition task. We have shown previously that without noise, two interneurons are sufficient for this task, but such networks are fragile to even the slightest variation of the timing of inputs [39]. In contrast, networks with three interneurons can be evolved to recognize patterns consisting of three stimuli in the presence of noise, and they are robust to a change of neuronal parameters or duration of intervals of silence between the stimuli $[38,40]$. In this work, we use the same model of noise (on the membrane voltage) as previously; while its level is biologically realistic, and so including it adds to the biological plausibility of our model, our primary concern is to aid in the evolution of networks that can maintain their states (a form of memory) even as the intervals between stimuli are hugely increased when testing the evolved network. One of the original contributions of this paper is that evolving the networks both in the presence of noise and the variation of intervals between stimuli biases the networks towards those that can maintain their states.

We observe that a variety of network topologies resulting from an artificial evolutionary process can perform the same computational task [38-40]. This is also the case for biological networks [18,22]. By using artificial evolution, we are able to find the commonalities between the networks that can accomplish simple, but not trivial, computational tasks.

The recognition of temporal pattern requires temporal storage of the stimulus or delays [16,30-33]. Since our networks are very small, delays caused by synaptic delays are minimal. The main contribution of this paper is that the crucial connections that maintain the network state and memory in the presence of variable silent intervals are self-excitatory loops (autapses), which sheds new light on the importance of these connections that are commonly found in biological neural systems $[26,36]$. Furthermore, persistent spiking activity in response to short sensory input is common in all areas of brain [17] which perhaps is responsible for keeping short-term memory in accumulating tasks [27].

\section{Methods}

Each network in our model is encoded in a linear genome, and consists of three inputs, three interneurons, and one output neuron [38-40]. Inputs are not allowed to connect to the output neuron directly and only interneurons can have self-loops. A fully connected network with this structure can have up to 21 connections (up to nine connections from inputs to interneurons, six connections between the interneurons, three self-loops, and three connections from the interneurons to the output neuron). 
Each input is dedicated to one signal (stimulus type), denoted as A, B and C. The interneurons and output neuron are modelled using Euler integration with $1 \mathrm{~ms}$ steps of the differential equations for adaptive exponential integrate and fire neurons [20]; we use the same parameter values as in [38-40]; these values result in tonic spiking in response to constant input current. Since this study focuses on the effect of network connectivity, the neuronal parameters are kept constant (allowing them to evolve would hugely increase the search space of the artificial evolutionary process). To simulate noisy synaptic background at a biologically realistic level $[2,7,9,21]$, we add a random value taken from a normal distribution with standard deviation $2 \mathrm{mV}$ and mean 0 to the membrane potential of each neuron at every $1 \mathrm{~ms}$ simulation step. When a neuron receives a spike the excitatory $g_{E}$ or inhibitory $g_{I}$ conductance is updated by the connection weight multiplied by the respective conductance gain. The value of the excitatory and inhibitory gain is $7 \mathrm{nS}$.

The task of the networks is to recognize three signals in a particular order $(\mathrm{ABC})$ in a continuous random sequence (...BCACACCABCACBAC...), in which all signals appear with equal probability, and thus the correct patterns take up about $10 \%$ of time. To generate a variety of solutions, we use a genetic algorithm with a population of 300 individuals, with 100 independent runs for each of the two settings: in the first setting signals are followed by a constant interval of silence (16 ms), in the second setting the intervals vary, with a uniform distribution between 16 and $32 \mathrm{~ms}$ (in previous work, [38, 40], we used noise on the membrane potential, but did not vary the interval of silences). In both settings the length of a each signal is $6 \mathrm{~ms}$. We use the same genetic operators as in [40]; they can result in changes of weights, deletion and addition of edges (synapses) and the nodes (neurons) in the network (through deletion and duplication, respectively, of consecutive elements in the linear genomes; however, the maximum size of the network was limited as described above).

Each individual in the population in each generation is evaluated on six sequences. Four out of these six sequences are generated randomly with equiprobable occurrence of three signals A, B and C; the remaining two sequences consist of four concatenated patterns in random order: $\mathrm{ABC}$ and three patterns that are hard to distinguish from this target (ABA, ABB, and BBC). The fitness function $[38,40]$ rewards networks in which the output neuron spiked (at least once) in the correct intervals, and did not spike in the incorrect intervals: $f_{\text {fitness }}=1-R+4 P$, where $R$, reward $(P$, penalty) is the fraction of correct (incorrect) intervals in which output spiked. $P$ is multiplied by 4 in this formulation because its denominator is much larger than the numerator for the networks that have correct performance or are close to it (when the input sequence is random, $90 \%$ of intervals are incorrect). The correct intervals are those that start at the onset of the last signal $(\mathrm{C})$ of the correct pattern $(\mathrm{ABC})$ and end with the end of the silence that follows. Similarly, incorrect intervals start at the onset of each signal that is not $\mathrm{C}$ in $\mathrm{ABC}$. Both correct and incorrect intervals last either 22 $\mathrm{ms}$ or, in the setting with variable silence intervals, $22-38 \mathrm{~ms}$. 
The false discovery rate (FDR) of the network is defined as the number of incorrect intervals in which output spiked divided by the sum of both incorrect and correct intervals in which the output spiked. The true positive rate (TPR) of the network is the same as $R$. We define a champion in a run as a perfect recognizer if its TPR is above 0.99 and FDR below 0.01 for the settings (constant or variable interval of silence between the signals) under which a champion was evolved.

In order to simplify the network analysis, we use pruning of superfluous edges in the network. Our pruning algorithm removes excessive connections in two steps in a loop: (i) a random connection is removed for testing; (ii) if $\mathrm{TPR}<0.95$ or FDR $>0.05$, the connection is reinstated and labelled as vital; the loop is terminated when all the connections are labelled as vital.

\section{Results and Discussion}

Out of 100 independent runs in the presence of noise on membrane potential but with constant interval of silence between the signals, 15 ended with champions that were perfect recognizers; when in addition to noise the intervals of silence varied during evolution, the yield was $12 \%$.

Even though our artificial evolutionary process allows for deletion of nodes (neurons) in the network, none of the perfect recognizers had less than three interneurons. In addition, even though pruning can result in a disconnection of a node, no network ended up with less than three interneurons after pruning. Perfect recognizers evolved only with noise had slightly more (19.20 on average; Table 1) edges than the perfect recognizers evolved also with variation of silences (18.83). This difference persisted after pruning (14.26 and 13.08 edges, respectively). None of these differences were statistically significant.

We tested both the evolved and pruned networks on a random sequence with 100,000 signals and $100 \mathrm{~ms}$ intervals of silence between signals. Our results (Table 1) show, firstly, that evolving the networks with both noise and variation of silences resulted in more perfect recognizers that can keep memory (11 out of 12) than for evolving only with noise (4 out of 15). Secondly, all these $11+4=15$ perfect recognizers kept memory also after pruning, demonstrating that the removed connections are unnecessary not only for recognizing the pattern but also for keeping memory.

Interestingly, while the perfect recognizers that kept memory had fewer selfexcitatory loops after pruning (all had 2; Table 1) than the champions which did not keep memory (which on average had 2.42), the sum of weights of the selfexcitatory loops was significantly higher in recognizers that kept memory (mean sum 14.6 vs. $12.3 ; p=0.002$, one-sided Wilcoxon test). This suggests that the memory is maintained in these networks through self-excitation; we will explore this issue further below by analysing the mechanisms by which some networks keep memory while other fail to do so.

Based on the TPR and FDR with $100 \mathrm{~ms}$ intervals of silence (Table 1), we can divide the 27 perfect recognizers into four groups: (i) 15 memory-keepers 
Table 1. The number of edges and self-excitatory loops in perfect recognizers evolved with noise and constant (top, 15 champions) or variable (bottom, 12 champions) silences, and their robustness to the increase of silences to $100 \mathrm{~ms}$

\begin{tabular}{|c|c|c|c|c|c|c|c|c|c|c|c|c|c|c|c|}
\hline & 0 & 1 & 2 & 3 & 4 & 5 & 6 & 7 & 8 & 9 & 10 & 11 & 12 & 13 & 14 \\
\hline evolved edges & 19 & 21 & 20 & 19 & 18 & 19 & 19 & 18 & 21 & 16 & 20 & 20 & 19 & 20 & 19 \\
\hline edges after pruning & 10 & 14 & 15 & 13 & 11 & 14 & 16 & 15 & 16 & 16 & 17 & 16 & 15 & 13 & 13 \\
\hline self ex-loops & 1 & 3 & 2 & 3 & 2 & 2 & 2 & 2 & 3 & 3 & 3 & 2 & 2 & 2 & 2 \\
\hline $100 \mathrm{~ms}$ TPR & 0.02 & 0.98 & 0.98 & 0.97 & 0.96 & 0.95 & 0.99 & 0.55 & 0.99 & 0.00 & 0.00 & 0.00 & 0.96 & 0.99 & 0.96 \\
\hline $100 \mathrm{~ms} \mathrm{FDR}$ & 0.99 & 0.37 & 0.01 & 0.12 & 0.56 & 0.01 & 0.03 & 0.90 & 0.20 & 1.00 & 1.00 & 0.99 & 0.58 & 0.01 & 0.56 \\
\hline
\end{tabular}

Champions evolved in the presence of noise and variable (16-32 ms) intervals of silence

\begin{tabular}{ccccccccccccc}
\hline & 0 & 1 & 2 & 3 & 4 & 5 & 6 & 7 & 8 & 9 & 10 & 11 \\
\hline evolved edges & 19 & 21 & 20 & 19 & 20 & 18 & 16 & 20 & 18 & 19 & 18 & 18 \\
edges after pruning & 13 & 12 & 14 & 14 & 14 & 13 & 11 & 13 & 15 & 12 & 13 & 13 \\
self ex-loops & 2 & 2 & 2 & 2 & 3 & 2 & 2 & 2 & 2 & 2 & 2 & 2 \\
100ms TPR & 0.99 & 0.99 & 0.99 & 0.98 & 0.90 & 0.98 & 0.99 & 0.98 & 0.99 & 0.97 & 0.98 & 0.99 \\
100ms FDR & 0.04 & 0.01 & 0.01 & 0.01 & 0.40 & 0.04 & 0.01 & 0.01 & 0.04 & 0.01 & 0.02 & 0.01 \\
\hline
\end{tabular}

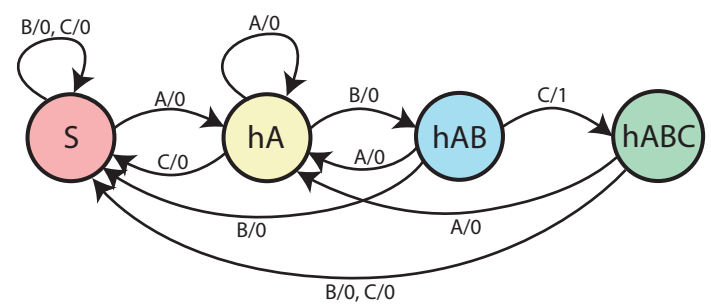

Fig. 1. Finite state transducer for recognizing ABC. The symbols above each arrow correspond to the input (A, B, C) and output (0: no spiking, 1: spiking of the output neuron).

(TPR remains high, and FDR low; evolved only with noise: number 2, 5, 6, 13; evolved with both noise and variation of silences: all except number 4), (ii) overrecognizers (TPR stays high, but FDR increases; evolved only with noise: 1, 3, 4, 8, 12, 14; evolved with both: number 4), (iii) wrong-recognizers (low TPR, high FDR; evolved only with noise: number $0,7,10,11$ ), (iv) mute networks (champion 9 evolved only with noise), for which long intervals of silence between signals result in only noise driven activity (negligible) of the output neuron. To illustrate what allows for both recognition and memory, we first analyse one memory-keeper evolved with both noise and variation of silences (champion 6; Fig. 2), one over-recognizer (champion 4; Fig. 3), one wrong-recognizer (champion 7 ; Fig. 4), and the mute network (champion 9; Fig. 5). The champions 4,7 and 9 fail when silences are $50 \mathrm{~ms}$ long for the same reasons they fail with 100 ms silences; we use $50 \mathrm{~ms}$ in the figures to keep them compact. While the activities of the pruned networks that fail are slightly different from the activities of the evolved networks, they fail for the same reasons; we will present only the analysis of the pruned networks for simplicity. 


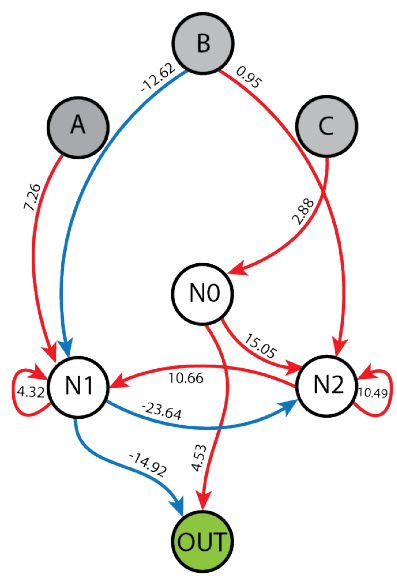

(a)

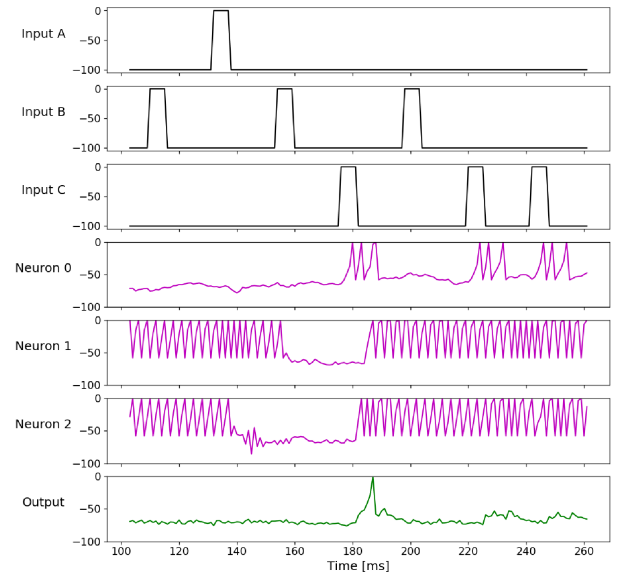

(b)

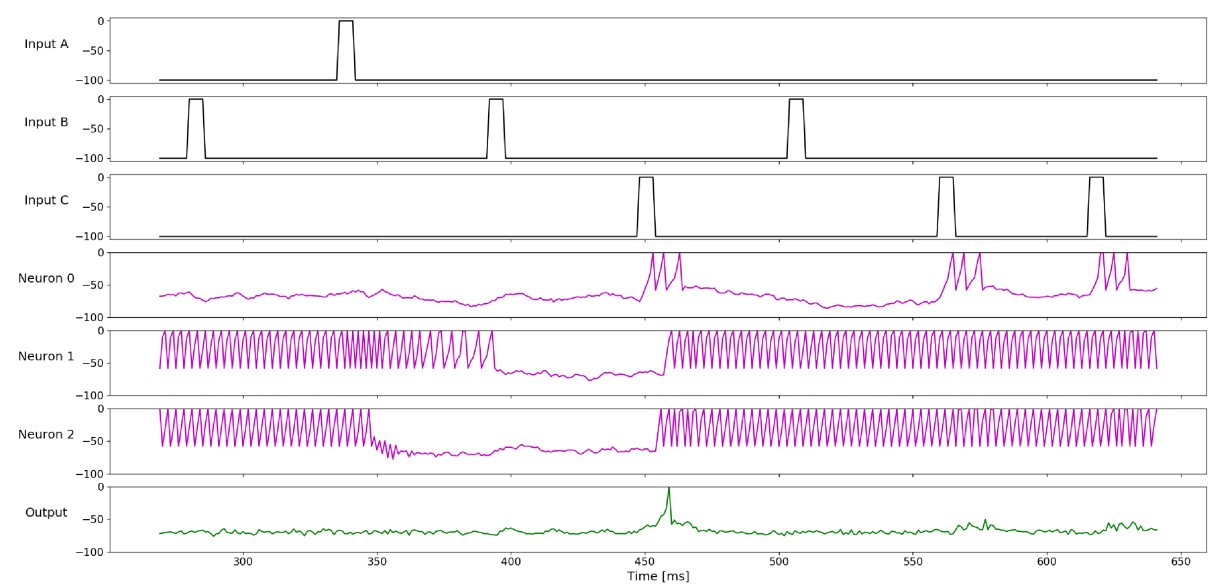

(c)

Fig. 2. Champion 6 evolved with both noise and variation of silences. The activity of the pruned network (a) is shown for short (16 ms; b), and long (50 ms; c) silences, which indicates that this champion is a memory-keeper.

In order to analyse the transitions of the network, we will use the paradigm we proposed previously, based on mapping the network states onto the states of a finite state transducer (FST) [38-40]. A FST, a formal computational model [28], is frequently used for analysing computations on time series performed in an online manner (that is, constantly producing an output for a continuous input). The minimal FST for recognizing a pattern with three symbols has four states (Fig. 1).

In the case of our memory-keeper (Fig. 2), the activity of input A, because of its excitatory connection to N1, makes N1 spike; this spiking continues thanks to the excitatory self-loop, while N0, N2, and output remain silent. Thus, we can denote the state hA (for 'had A', Fig. 1) as LHL, where L (H) means low 
(high) activity for interneurons in the order (N0, N1, N2). The state hAB (when the network receives $\mathrm{B}$ after $\mathrm{A}$, for 'had $\mathrm{AB}$ ') can be denoted as LLL, and the state hABC ('had ABC') as LHH. The only difference between the start state $(\mathrm{S})$ and hABC is the intermittent activity of the output. Thus, the state of the network while waiting for the last signal in the pattern (state hAB) is maintained passively (all interneurons are silent), while the other states, hA and $\mathrm{hABC} / \mathrm{S}$, are maintained by the self-excitatory loops on $\mathrm{N} 1$ and $\mathrm{N} 2$. In hA, only N1 is continuously active; in $\mathrm{hABC} / \mathrm{S}$, both N0 and N1 are. The inhibitory connection from $\mathrm{N} 1$ to the output ensures that receiving a signal $\mathrm{C}$ will cause the output to spike (after N0 spikes) only when N1 - and N2, which activates $\mathrm{N} 1$ - are silent. The inhibitory connection from input B to N1 is necessary for N1 to cease its activity in hAB, but this does not happen when $\mathrm{N} 2$ is active (so when network is in the state $\mathrm{hABC}$ or $\mathrm{S}$, it goes to $\mathrm{S}$ after receiving $\mathrm{B}$ ). The inhibitory connection from $\mathrm{N} 1$ to $\mathrm{N} 2$ is necessary for the transition from hABC/S to hA (higher frequency of N1 shuts down N2). Finally, the weak excitatory connection from $\mathrm{B}$ to $\mathrm{N} 2$ is necessary to ensure that when $\mathrm{N} 2$ is silent (hAB), receiving a $\mathrm{B}$ would not silence both $\mathrm{N} 1$ and $\mathrm{N} 2$; indeed, when this connection is removed, the output wrongly spikes after receiving $\mathrm{ABBC}, \mathrm{ABBBC}$, etc. (the network recognizes the regular expression $\mathrm{AB}^{+} \mathrm{C}$, not just $\mathrm{ABC}$ ).

While we only describe one memory-keeper here, in all such networks analysed so far, the state hAB is represented by LLL (and thus the networks will spike when they are initiated with no activity and receive just a $\mathrm{C}$ ), and the networks maintain two states stably: hA and hABC/S (which differ only by the short-term activity of the output, triggered by the transition from hAB to $\mathrm{hABC}$ ). Since the over-recognizer we have chosen for analysis (Fig. 3) shares its topology with the memory-keeper (Fig. 2), both recognize ABC correctly in the same fashion when the silence intervals are short-when the network receives A, it goes to the state LHL, when B follows, to LLL, and when C follows, to LHH. However, when the silences are long, the activity of N2 in LHH dies out (because the N2 self-excitatory loop is weak), and the network goes to LHL - the same as $\mathrm{hA}$. When B is received in this state, all activity ceases (state LLL). If the next signal is C, the output neuron spikes, wrongly. This leads to a high FDR - the network recognizes the pattern $\mathrm{BC}$ when the intervals are long.

The analysis of the network activity of the wrong-recognizer (Fig. 4) reveals that the recognition when the intervals of silence are short depends on transitions from one unstable state to another. We can see that when the intervals are long, the $\mathrm{S}$ state (HHH) is stable, and maintained by the strong self-excitatory loop on N1. When A is received, N0 speeds up, and inhibits N1 (the networks goes to the state HLH). If the silence continues, the network goes to the state LLH (N0 does not have any self-loop), and then LLL (the self-loop of N2 is too weak to maintain its activity for long). If $\mathrm{C}$ is received at this point, $\mathrm{N} 1$ spikes, and without inhibition from N2, the output spikes, leading to the recognition of AC. When the intervals are short, the network transitions along the same trajectory when it recognizes $\mathrm{ABC}$, but much quicker-when it is still in the state HLH after receiving $\mathrm{A}$, the arrival of a $\mathrm{B}$ pushes it to LLH, which can relax to LLL 


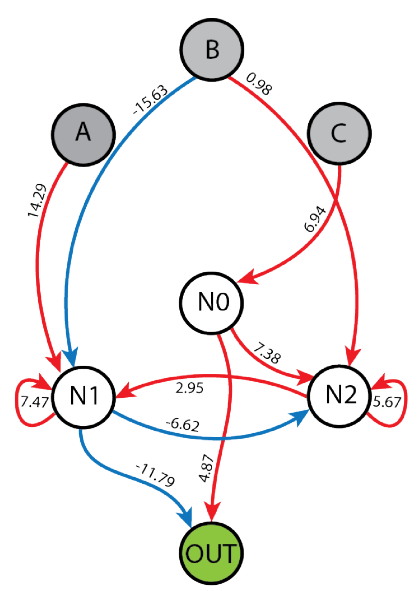

(a)

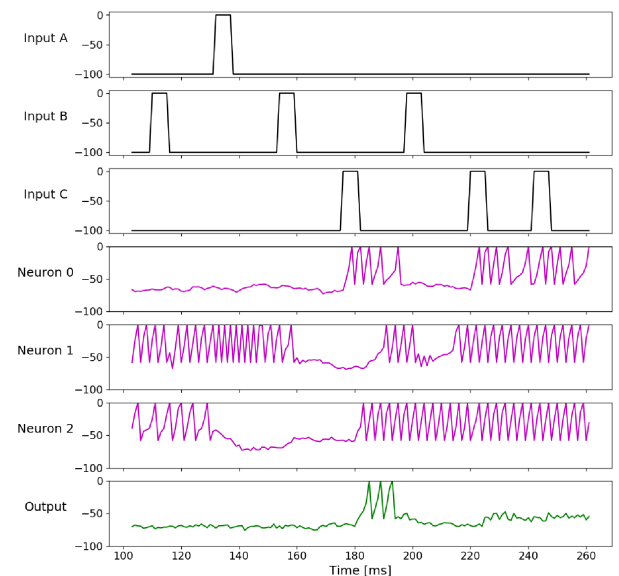

(b)

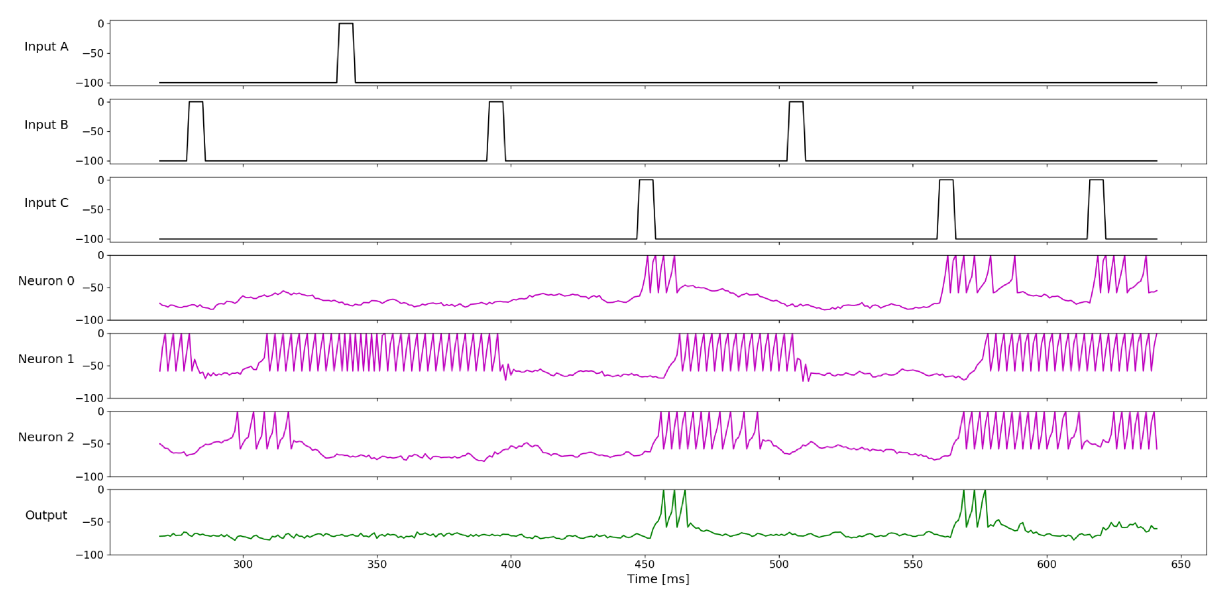

(c)

Fig. 3. Champion 4 evolved with noise and constant silences. The activity of the pruned network (a) is shown for short (16 ms; b), and long (50 ms; c) silences, for which this champion behaves as an over-recognizer.

in time to release the output from the inhibition from N2 when the spike of N1, induced by receiving a $\mathrm{C}$, arrives. Any Bs that do not follow an A after a short interval of silence, and any $\mathrm{Cs}$, cause the network to go the stable state $\mathrm{HHH}$.

Finally, in champion 9 evolved with constant short intervals of silence, the recognition of $\mathrm{ABC}$ crucially depends on the network being in a particular unstable state when the network receives a $\mathrm{C}$ after having received the pattern $\mathrm{AB}$ - to activate the output, N2 (active after $\mathrm{C}$ is received) needs to spike fast; and for $\mathrm{N} 2$ to spike fast, $\mathrm{N} 2$ needs to receive also the activation of N1. However, N1 cannot spike too frequently because it inhibits the output. In addition, N0 (which also inhibits the output) needs to be inactive. This particular state can only be achieved if the network first reaches the state HLL (which is sta- 


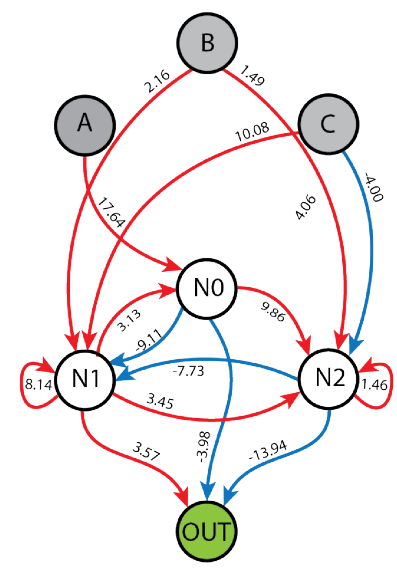

(a)

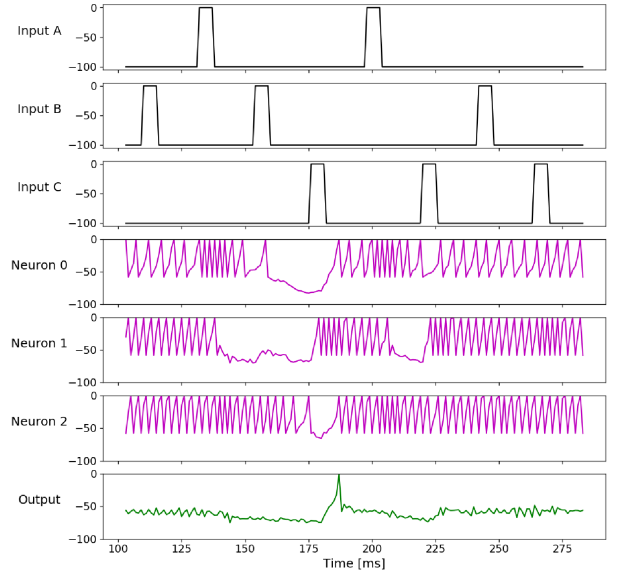

(b)

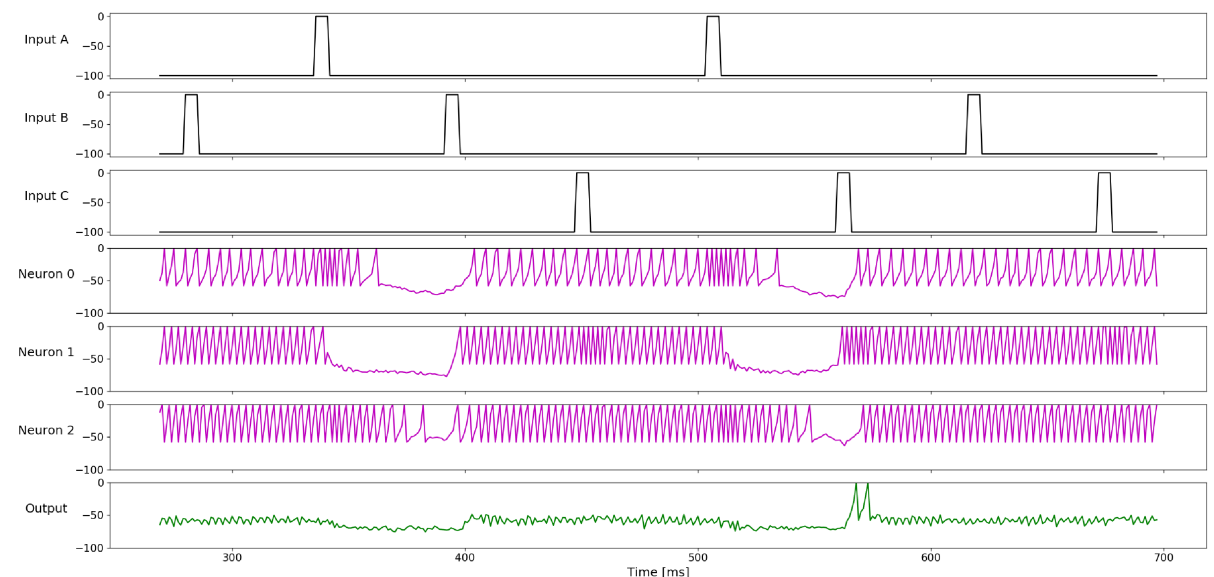

(c)

Fig. 4. Champion 7 evolved with noise and constant silences. The activity of the pruned network (a) is shown for short (16 ms; b), and long (50 ms; c) silences, for which this champion behaves as a wrong-recognizer.

ble thanks to the self-excitatory loop on N0). With short silences, this state is reached after receiving an $\mathrm{A}$. When the silence is long, the right conditions for the output to spike never occur - even though there are times when N1 spikes slowly with N0 inactive, N2 never spikes frequently enough at that time to drive the output to spike. Moreover, even though N1 also has a self-excitatory loop, its activity cannot be sustained for long when N0 (which inhibits N1) is active, and because N1 activates N2, which in turn activates N0, N1 can only spike slowly.

Our experimental setup did not impose the Dale's rule [4] on the evolved networks. This is because our preliminary experiments showed that imposing the rule would require permitting more (roughly double) interneurons during 


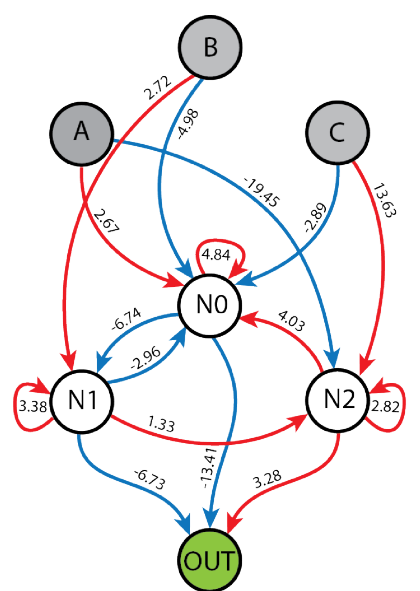

(a)

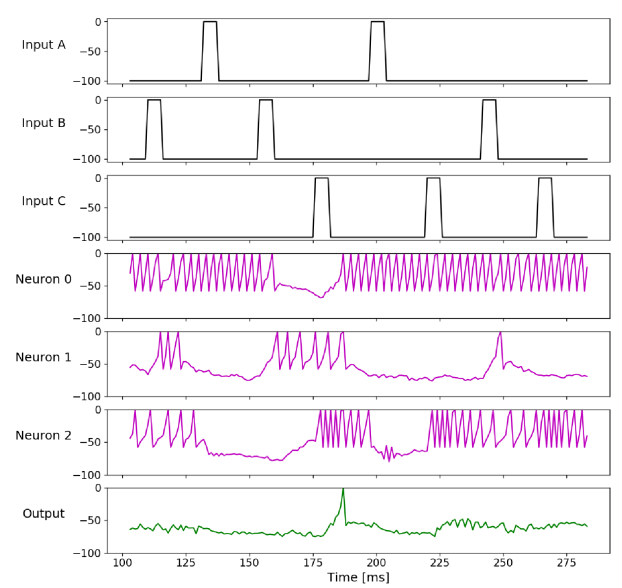

(b)

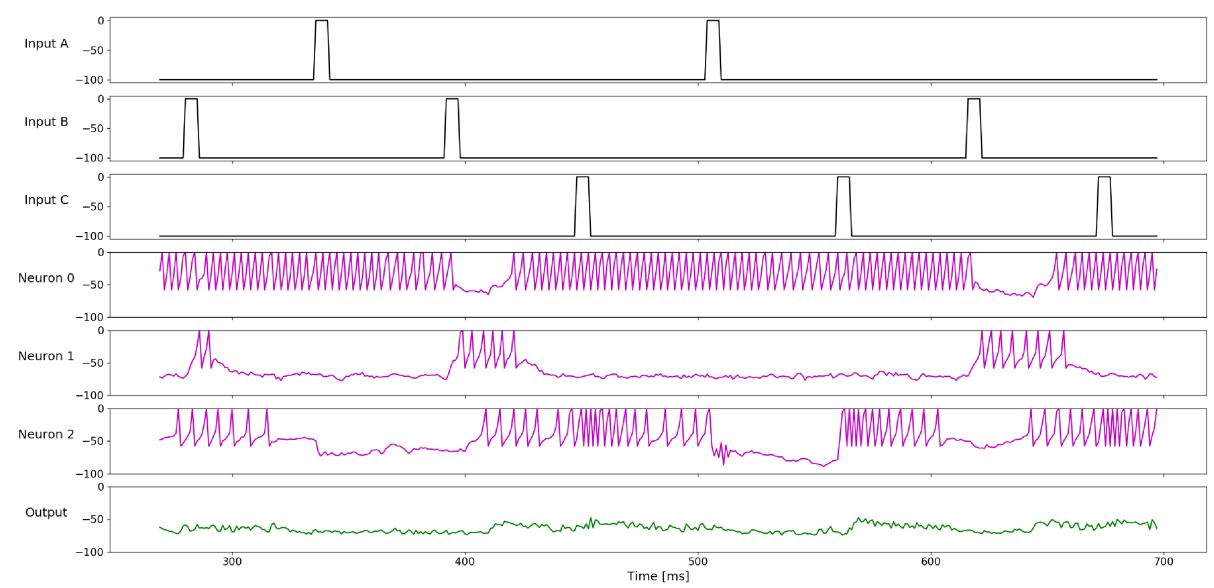

(c)

Fig. 5. Champion 9 evolved with noise and constant silences. The activity of the pruned network (a) is shown for short (16 ms; b), and long (50 ms; c) silences, for which this champion behaves as a mute network.

evolution, increasing the search space. All of the networks we analysed had at least one interneuron which had both excitatory and inhibitory connections to other neurons in the network, even after pruning the superfluous connections. However, once a network is evolved, it is straightforward to transform it to a network that conforms to the Dale's rule. This can be done by splitting a neuron that violates the rule into two new neurons, one excitatory and one inhibitory (Fig. 6). Pruning superfluous connections from the network leads to fewer neurons for which such splitting is necessary. Both new neurons receive the same inputs (with the same weights) as the original neuron. The weights of the outgoing connections are also maintained. If an excitatory self-loop is present, it 
is maintained with the same weight for the new excitatory neuron, and a new excitatory connection with the same weight is created from the new excitatory to the new inhibitory neuron (an analogous operation can be made for an inhibitory self-loop, should one exist). The formation of this new connection could, in principle, affect the functionality of the network, as it creates an additional synaptic delay. However, the networks evolved with noise can be expected to be robust to such a perturbation. On the other hand, creating two noisy neurons instead of one creates, in principle, a more noisy network. The performance of the networks analysed in this paper was not affected by the transformation detailed here. This applies, in particular, to champion 6 , the memory-keeper, who has only one neuron (N1) that violates the rule (Fig. 2,6).

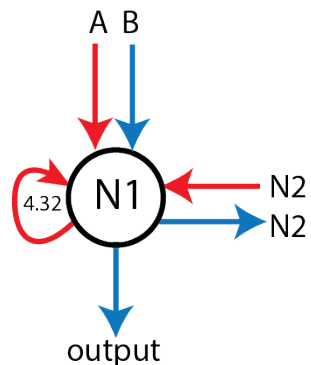

(a)

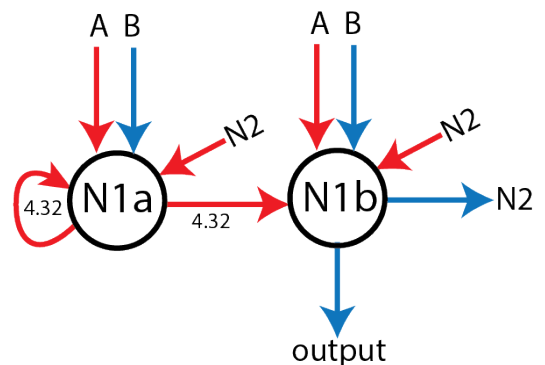

(b)

Fig. 6. Splitting a neuron that violates the Dale's rule in order to create the network that conforms to the rule. The single interneuron, N1, that violates the rule in champion 6 (Fig. 2), with two inhibitory outputs and one excitatory self-loop (a), can be split into two new neurons, one excitatory and one inhibitory (b).

\section{Conclusions and Future Work}

Our analysis of very small networks evolved to recognize simple temporal patters reveals that many connections in these networks can be removed without impairing the performance of the network. Such pruning allows for a much easier understanding of the mechanisms in which the networks accomplish this minimally cognitive task.

For the networks analysed in this paper, these mechanisms depend crucially on the presence of strong self-excitatory loops, necessary for both the pattern recognition and maintenance of network state - a form of memory. Our results indicate that to recognize a pattern consisting of three symbols with state maintenance, the networks need to consist of at least three interneurons (with one output), and need to have two self-excitatory loops with the weights sufficient to maintain the network states. Our analysis of the activity of the networks that keep memory, by mapping the network states on the states of an FST, shows that all the perfect recognizers that maintain the states of the network represent 
the state before the arrival of the final symbol by inactivity of the network (a state that does not need to be maintained actively). In all these networks, the accepting state differs from the start state only by the intermittent activity of the output (triggered on the transition to this state from the state of inactivity). This state is maintained actively, and so is the state reached after receiving the first symbol in the pattern.

Our analysis of the networks that fail to maintain the memory correctly reveals the following preliminary insights. With long intervals of silence between the signals, over-recognition happens when the network does not maintain the start state or the state after the correct pattern is recognized (which may be the same), but instead over the long interval of silence transitions to the same state as the one reached after receiving the first symbol in the pattern. With long silences, such networks continue to recognize the correct pattern but start to recognize wrong patterns. On the other hand, the networks that cannot maintain the other states with long silences can either stop recognizing the correct pattern while recognizing wrong ones, or become completely mute.

We show that the perfect recognizers that keep memory function essentially follow the paradigm of an FST. Previous work on creating finite state machines based on recurrent spiking neural networks [19, 24,35] considered large multilayer networks. Here we show, essentially, a method to obtain very small networks that work a finite automata using artificial evolution. In future work, we plan to investigate the limits of the length of the temporal patterns for which prefect recognizers that keep memory can be evolved, and how many self-excitatory loops are necessary in such recognizers, for both shorter and longer patterns.

In the work reported here and previously [38-40], we have allowed only for the topology to change. In principle, the neuronal parameters could also be evolved, but this would hugely increase the search space. However, other spiking behaviours (for example, bursting) of the neurons in the network could perhaps lead to different classes of solutions. We plan to explore this issue in our future work using two approaches: (i) allowing a discrete change of the behaviour of each neuron in the network during evolution (for example, from tonic spiking to bursting, a change of the values of several parameters in one step), (ii) by exploring if the solutions change when all the neurons in the network have the same behaviour (different than used here). We could also modify our model of artificial evolution to allow for a more efficient search for the solutions; a different evolutionary model might possibly also lead to different classes of solutions.

Furthermore, we plan to revisit the question of robustness of the evolved networks to changes of parameters and synaptic weights. We also plan to investigate if other models of noise (such as an Ornstein-Uhlenbeck process, commonly used in computational neuroscience), variation of silences or neuronal parameters during evolution will influence the types of solutions, their evolvability and robustness.

Acknowledgements. This work was supported by the Polish National Science Center (project EvoSN, UMO-2013/08/M/ST6/00922). MY acknowledges the 
support of the KNOW RNA Research Center in Poznan (No. 01/KNOW2/2014) and POWR.03.02.00-00-I006/17.

\section{References}

1. Ahissar, E., Arieli, A.: Figuring space by time. Neuron 32, 185-201 (2001)

2. Anderson, J.S., Lampl, I., Gillespie, D.C., Ferster, D.: The contribution of noise to contrast invariance of orientation tuning in cat visual cortex. Science 290, 1968$1972(2000)$

3. Bialek, W., Rieke, F., van Steveninck, R.R.d.R., Warland, D., et al.: Reading a neural code. In: Neural Information Processing Systems. pp. 36-43 (1989)

4. Burnstock, G.: Autonomic neurotransmission: 60 years since sir Henry Dale. Annual Review of Pharmacology and Toxicology 49, 1-30 (2009)

5. Decharms, R.C., Zador, A.: Neural representation and the cortical code. Annual Review of Neuroscience 23, 613-647 (2000)

6. Destexhe, A., Rudolph, M., Fellous, J.M., Sejnowski, T.: Fluctuating synaptic conductances recreate in vivo-like activity in neocortical neurons. Neuroscience 107, 13-24 (2001)

7. Destexhe, A., Paré, D.: Impact of network activity on the integrative properties of neocortical pyramidal neurons in vivo. Journal of Neurophysiology 81, 1531-1547 (1999)

8. Faisal, A.A., Selen, L.P., Wolpert, D.M.: Noise in the nervous system. Nature Reviews Neuroscience 9, 292-303 (2008)

9. Finn, I.M., Priebe, N.J., Ferster, D.: The emergence of contrast-invariant orientation tuning in simple cells of cat visual cortex. Neuron 54, 137-152 (2007)

10. Florian, R.V.: Biologically inspired neural networks for the control of embodied agents. Center for Cognitive and Neural Studies (Cluj-Napoca, Romania), Technical Report Coneural-03-03 (2003)

11. Gerstner, W., Kempter, R., van Hemmen, J.L., Wagner, H.: A neuronal learning rule for sub-millisecond temporal coding. Nature 383, 76-78 (1996)

12. Huxter, J., Burgess, N., O'keefe, J.: Independent rate and temporal coding in hippocampal pyramidal cells. Nature 425, 828-832 (2003)

13. Jacobson, G., Diba, K., Yaron-Jakoubovitch, A., Oz, Y., Koch, C., Segev, I., Yarom, Y.: Subthreshold voltage noise of rat neocortical pyramidal neurones. The Journal of Physiology 564, 145-60 (2005)

14. Joris, P., Yin, T.: A matter of time: internal delays in binaural processing. Trends in Neuroscience 30, 70-78 (2007)

15. Laurent, G.: Dynamical representation of odors by oscillating and evolving neural assemblies. Trends in Neurosciences 19, 489-496 (1996)

16. Maex, R., Steuber, V.: The first second: Models of short-term memory traces in the brain. Neural Networks 22, 1105-1112 (2009)

17. Major, G., Tank, D.: Persistent neural activity: prevalence and mechanisms. Current Opinion in Neurobiology 14, 675-684 (2004)

18. Marder, E.: Variability, compensation, and modulation in neurons and circuits. Proceedings of the National Academy of Sciences USA 108, 15542-15548 (2011)

19. Natschläger, T., Maass, W.: Spiking neurons and the induction of finite state machines. Theoretical Computer Science 287, 251-265 (2002)

20. Naud, R., Marcille, N., Clopath, C., Gerstner, W.: Firing patterns in the adaptive exponential integrate-and-fire model. Biological Cybernetics 99, 335-347 (2008) 
21. Paré, D., Shink, E., Gaudreau, H., Destexhe, A., Lang, E.J.: Impact of spontaneous synaptic activity on the resting properties of cat neocortical pyramidal neurons in vivo. Journal of Neurophysiology 79, 1450-1460 (1998)

22. Prinz, A.A., Bucher, D., Marder, E.: Similar network activity from disparate circuit parameters. Nature Neuroscience 7, 1345-1352 (2004)

23. Rieke, F., Warland, D., de Ruyter van Steveninck, R., Bialek, W.: Spikes: Exploring the Neural Code. MIT Press (1999)

24. Rutishauser, U., Douglas, R.J.: State-dependent computation using coupled recurrent networks. Neural Computation 21, 478-509 (2009)

25. S Isaacson, J.: Odor representations in mammalian cortical circuits. Current Opinion in Neurobiology 20, 328-31 (2010)

26. Saada, R., Miller, N., Hurwitz, I., J Susswein, A.: Autaptic excitation elicits persistent activity and a plateau potential in a neuron of known behavioral function. Current Biology 19, 479-84 (2009)

27. Seung, H.S., Lee, D.D., Reis, B.Y., Tank, D.W.: The autapse: A simple illustration of short-term analog memory storage by tuned synaptic feedback. Journal of Computational Neuroscience 9, 171-185 (2000)

28. Sipser, M.: Introduction to the Theory of Computation. International Thomson Publishing (1996)

29. Stacey, W., Durand, D.: Stochastic resonance improves signal detection in hippocampal neurons. Journal of Neurophysiology 83, 1394-1402 (2000)

30. Steuber, V., De Schutter, E.: Rank order decoding of temporal parallel fibre input patterns in a complex Purkinje cell model. Neurocomputing 44-46, 183-188 (2002)

31. Steuber, V., J. Willshaw, D.: Adaptive leaky integrator models of cerebellar Purkinje cells can learn the clustering of temporal patterns. Neurocomputing 26-27, 271-276 (1999)

32. Steuber, V., Willshaw, D.: A biophysical model of synaptic delay learning and temporal pattern recognition in a cerebellar Purkinje cell. Journal of Computational Neuroscience 17, 149-164 (2004)

33. Steuber, V., Willshaw, D., Ooyen, A.V.: Generation of time delays: Simplified models of intracellular signalling in cerebellar Purkinje cells. Network Computation in Neural Systems 17, 173-191 (2006)

34. Thorpe, S., Fize, D., Marlot, C.: Speed of processing in the human visual system. Nature 381, 520-522 (1996)

35. Tino, P., J S Mills, A.: Learning beyond finite memory in recurrent networks of spiking neurons. Neural Computation 18, 591-613 (2005)

36. Wang, C., Guo, S., Xu, Y., Ma, J., Tang, J., Alzahrani, F., Hobiny, A.: Formation of autapse connected to neuron and its biological function. Complexity 2017, 1-9 (2017)

37. Wiesenfeld, K., Moss, F.: Stochastic resonance and the benefits of noise: From ice ages to crayfish and squids. Nature 373, 33-36 (1995)

38. Yaqoob, M., Wróbel, B.: Robust very small spiking neural networks evolved with noise to recognize temporal patterns. In: ALIFE 2018: Proceedings of the 2018 Conference on Artificial Life - MIT Press. pp. 665-672 (2018)

39. Yaqoob, M., Wróbel, B.: Very small spiking neural networks evolved to recognize a pattern in a continuous input stream. In: 2017 IEEE Symposium Series on Computational Intelligence (SSCI) - IEEE. pp. 3496-3503 (2017)

40. Yaqoob, M., Wróbel, B.: Very small spiking neural networks evolved for temporal pattern recognition and robust to perturbed neuronal parameters. In: Artificial Neural Networks and Machine Learning - ICANN. pp. 322-331 (2018) 